\title{
Mesut Yilmaz* \\ Der Eintritt der Türkei in die EU aus türkischer Sicht
}

\author{
Die Wendung der Türkei nach Westen
}

Die ganze Geschichte hindurch haben sich die Türken von Osten nach Westen bewegt und sich immer am Westen ausgerichtet. In der jüngeren Vergangenheit geht der türkische Aufbruch, westliche Institutionen zu übernehmen, auf eine 200-jährige Geschichte zurück.

In der Spätphase des Osmanischen Reiches wurde die Lösung, den Zusammenbruch des Imperiums abzuwenden, in westlich orientierten Reformen gesehen. Die Türkei, die sich in Verwaltung, Militärwesen, Bildung und Recht den Westen zum Vorbild wählte, unternahm seit Beginn des 19. Jahrhunderts grundlegende Reformen. Dieser Prozess, der seine Konturen mit dem »Tanzimat Fermani « ${ }^{1}$ gewann, ist nach wie vor nicht abgeschlossen. Selbst als am Ende des Ersten Weltkrieges, in den das Osmanische Reich an der Seite Deutschlands eintrat, das Reich durch die europäischen Staaten vernichtet wurde, trat keine Unterbrechung dieses Prozesses ein - im Gegenteil: er beschleunigte sich noch. Atatürk, dessen Position wir als »Westorientierung trotz des Westens « charakterisieren können, hat einen westlichen Entwicklungsstil als unumkehrbaren Weg festgelegt.

Der Grund, der das Osmanische Reich, einstmals das größte und mächtigste Imperium der Welt, in den Untergang trieb, lag im Verlust seiner Fähigkeit, sich Neuerungen zu öffnen. Um die Wiederholung dieses Fehlers zu verhindern, hat Atatürk die neue Republik auf Prinzipien errichtet, die Entwicklung und Fortschritt gegenüber offen sind. Das Kalifat, das arabische Alphabet, der islamische Kalender und das auf dem Islam beruhende Rechtssystem wurden aufgehoben und an seiner Stelle ein laizistisches System gegründet. Neben der Umgestaltung des Staates nach westlichem Muster wurden auch große Anstrengungen zu einer ebensolchen Wandlung der Gesellschaft unternommen. Atatürk setzte der türkischen Gesellschaft das Ziel, »den aktuellen Entwicklungsstand zu erreichen«, d. h. das Niveau Europas. Von jenen Tagen an haben sich türkische Intellektuelle und Politiker dieses Ziel zueigen gemacht.

* Früherer Ministerpräsident der Türkei, Gastprotessor an der Ruhr-Universität.

1 Mit diesem Sultans-Dekret wurde eine Staat und Gesellschaft umfassende Modernisierung der Türkei eingeleitet, die einen Meilenstein auf dem Weg des Übergangs des Osmanischen Reiches zum modernen türkischen Nationalstaat darstellt. Mert Toker, TürkAvrupa Günlüğ̈̈, Istanbul 2003, S. 142. 
Die 80-jährige Republik hat sich stets als Teil des Westens angesehen und in der Gemeinschaft der westlichen Staaten ihren Platz eingenommen. Nach dem Zweiten Weltkrieg hat die Türkische Republik ihre außenpolitische Präferenz ebenfalls zugunsten des Westens gesetzt und sich für ein gemeinsames Vorgehen mit dem Westen entschieden ${ }^{2}$. Angefangen mit der NATO ist die Türkei allen europäischen Institutionen beigetreten. Aus ihrer Westorientierung hat sich als natürliche Schlussfolgerung auch 1959 der Antrag zur damaligen Europäischen Wirtschaftsgemeinschaft ergeben.

\section{Das türkische EU-Abentener}

Seit die Türkei im Jahr 1959 ihren Antrag auf Aufnahme in die Europäische Union gestellt hat, sind 45 Jahre vergangen. Es gibt kein anderes Land, das 45 Jahre lang vor den Pforten der EU auf Einlass wartete. Einer der Meilensteine dieser 45-jährigen Reise ist der Ankara-Vertrag von 1963. Mit diesem Vertrag wurde der Türkei der Status eines Partners verliehen. Im gleichen Vertrag wurde nach einer 22-jährigen Übergangszeit die Verwirklichung einer Zollunion vorgesehen und als Endziel die Vollmitgliedschaft genannt. 1986 stellte die Türkei ihren Antrag auf Vollmitgliedschaft und seit 1996 ist sie zu einer Zollunion mit der EU übergegangen. Als beim EU-Gipfel von Luxemburg für die übrigen 12 Beitrittskandidaten die Aufnahme der Beitrittsverhandlungen beschlossen wurde, schlug man der Türkei einen »besonderen Status « vor. Die Türkei hat diesen Vorschlag abgelehnt und ihren politischen Dialog mit der EU ausgesetzt. Schließlich wurde 1999 beim Gipfel von Helsinki endlich der Kandidatenstatus der Türkei anerkannt und beschlossen, dass, wenn sie die Kopenhagener Kriterien erfüllt, Beitrittsverhandlungen aufgenommen werden. Beim Kopenhagener Gipfel Ende des Jahres 2002 wurde festgelegt, die Frage der Aufnahme von Beitrittsverhandlungen mit der Türkei spätestens bis Ende $2004 \mathrm{zu}$ entscheiden. Die 45-jährige Wartezeit hat bei der türkischen Bevölkerung zu Ungeduld geführt, eine Enttäuschung wird äußerst negative Folgen für die türkische Gesellschaft haben. Die Türkei geht davon aus, dass sie durch die seit 1999 vollzogenen tiefgreifenden Reformen die nötigen Anforderungen erfüllt, um die Beitrittsverhandlungen zu beginnen.

\section{Die Wabrnehmung der EU durch die türkische Gesellschaft}

In der Türkei durchgeführte Umfragen zeigen, dass die türkische Bevölkerung mit einer großen Mehrheit einer EU-Mitgliedschaft positiv gegenübersteht. Bevölkerungskreise mit recht unterschiedlicher Weltanschauung sehen sich im gemeinsamen Ziel der EU-Mitgliedschaft vereinigt. Dies deutet auf einen großen Wandel in der türkischen Öffentlichkeit hin.

2 Orhan Baskın (Hg.), Türk Dıș Politikası, Band I, Istanbul 2002.

ZfP 51.Jg. 2/2004 
Heute befürworten türkische Bürger aller politischen Richtungen, von den gemäßigten Islamisten bis zu Liberalen, von Sozialdemokraten bis zu gemäßigten Nationalisten, die Mitgliedschaft der Türkei in der EU. In diesem Rahmen lassen sich die Erwartungen des türkischen Volkes an eine EU-Mitgliedschaft wie folgt skizzieren:

- die Erreichung anhaltender wirtschaftlicher, politischer und gesellschaftlicher Stabilität,

- ein Leben in einem humanen Umfeld mit Geltung des Rechts und Wohlstand,

- die Vollendung der wirtschaftlichen Entwicklung durch die Anziehung ausländischer Investitionen.

Während der größte Teil der türkischen Bevölkerung die Mitgliedschaft in der EU wünscht, stellt sich ein nicht zu vernachlässigender Teil des Militärs und des Staatsapparats dem entgegen. Die grundlegenden Einstellungen und Einwände dieser Kreise lassen sich wie folgt zusammenfassen:

Die türkische EU-Integration wird dazu führen, dass diese in der EU eine "Satellitenstellung "innehmen wird³. Die Kontrolle über die Türkische Gesellschaft wird an die Fübrung der EU übergehen ${ }^{4}$. Die türkische EU-Integration wird zu ibrem Nachteil sein, die EU wird der Türkei nichts geben. Nicht zuletzt hat die Zollunion ebenso wie das Abkommen von Balta Limanı von 1838 die türkische Industrie in eine schwierige Lage gebracht, während die EU durch den Zugang zum türkischen Markt von 70 Millionen Menschen sowie die Möglichkeiten im Nahen Osten, Kaukasus und Mittelasien große geopolitische Gewinne erzieltes.

Jede Erweiterung der EU verstärkt ibre Schwäche aufgrund ibrer Multikulturalität, denn der Leitgedanke der EU ist nicht ein Leben in Multikulturalität, sondern eine Vereinigung auf der Grundlage gemeinsamer Werte und Eigenschaften zu erreichen. Aus diesem Grund wird unsere Mitgliedschaft verbindert oder zumindest sehr verzögert. Selbst wenn wir EU-Mitglied würden, blieben wir, anfgrund der Schwierigkeit, die gegen uns gehegten Vorurteile zu überwinden, immer Fremde. Man wird uns als die anderen "behandeln und damit fortfahren, mit unserer Ehre zu spielen ${ }^{6}$.

Sollte die Türkei EU-Mitglied werden, so wird man versuchen, das Land zu spalten $^{7}$.

Die dargestellten Einwände, die auf Erfahrungen der Vergangenheit und $\mathrm{Zu}-$ kunftsprojektionen zurückgehen, verfügen jedoch über nur geringe Unterstützung unter der Mehrheit der Bevölkerung.

3 Suat Ilhan, Avrupa Birliği'ne Neden Hayır, Istanbul 2000, S. 59.

4 Ebd., S. 53.

5 Ebd., S. 33, 93.

6 Ebd., S. 65-77.

7 Ebd., S. 138-139. 
Was die Untersuchungen zeigen

In der Türkei durchgeführte Meinungsumfragen stellen hinsichtlich der Wahrnehmung der EU durch die türkische Bevölkerung folgende Elemente in den Vordergrund:

»1. Die Befürwortung einer EU-Mitgliedschaft durch eine große Mehrheit der türkischen Öffentlichkeit hält an. Es ist bedeutsam, dass allen großen Ungewissheiten und schwerwiegenden Unstimmigkeiten beim Thema EU zum Trotz die Unterstïtzung auf diesem Niveau anbält.

2. Ein großer Teil der Öffentlichkeit glaubt, dass sich ibr Leben mit der EU-Mitgliedschaft positiv verändern wird.

3. Bei den positiven Veränderungen, die durch den EU-Beitritt ausgelöst werden, wird an wirtschaftliche Entwicklung, Demokratisierung, Verringerung der Korruption und die Freizügigkeit gedacht.

4. Jedoch haben die türkischen Bürger nicht die Absicht, sobald sich die Pforten der EU öffnen, auszuwandern. Die große Mebrheit möchte, wenn das Woblstandsniveau der Türkei wächst, ihr Leben im Land weiterführen.

5. Die türkische Öffentlichkeit zeigt keine religiös oder kulturell motivierte zurückweisende oder ablehnende Haltung gegenüber Europa oder europäischer Lebensweise.

6. Jedoch hat die türkische Öffentlichkeit den Eindruck, dass sich Europa der Türkei gegenüber nicht ausreichend aufrichtig verbält und die Türkei diskriminiert.

7. Die Öffentlichkeit bejabt unter der Bedingung, dass in Fragen der Verteidigung sowie der nationalen und religiösen Werte ausreichende Sensibilität anfgebracht wird, die Vereinigung mit der EU auf allen Gebieten und die Verfolgung gemeinsamer Politik.

8. Der Anteil derer, die glauben, dass die EU-Mitgliedschaft unsere Unabbängigkeit in Mitleidenschaft ziebt und zur Stärkung separatistischer oder radikaler religiöser Strömungen fübren könnte, ist sehr gering. « ${ }^{8}$

\section{Die Strategie, die Mitgliedschaft der Türkei so weit wie möglich hinauszuschieben}

Die eigentliche Befürchtung der Türkei ist nicht, im Jahr 2004 keinen Termin für die Beitrittsverhandlungen zu erhalten, sie entspringt vielmehr den Bemühungen von Kreisen in der EU, die Mitgliedschaft der Türkei so weit wie möglich zu verschieben. Diese Kreise haben es in 45 Jahren geschafft, eine Politiktradition zu schaffen, die türkische Mitgliedschaft auf jeder Stufe zu behindern oder, wenn sich die Gelegenheit ergibt, diese auch zu verhindern.

Die Aufnahme der Türkei in den Beitrittsprozess durch den Helsinki-Gipfel wurde in Europa heftiger diskutiert als in der Türkei, denn diese Entscheidung hat in Europa bei einigen Kreisen Verärgerung hervorgerufen. Ein Teil dieser Gruppen hat

8 TESEV, Türk Halkının AB Üyeliğine Bakıșı Araștırması, Istanbul 2002. 
ihre Befürchtungen auch offen zum Ausdruck gebracht: »Und wenn die Türkei sofort die erforderlichen Entscheidungen träfe und den Beginn der Beitrittsverhandlungen verlangte ... «Ein anderer Teil erklärte, dass die Mitgliedschaft der Türkei um 30 bis 40 Jahre hinausgeschoben werden müsse. Wieder ein anderer Teil ging davon aus, dass die Türkei die Bedingungen ohnehin nicht erfüllen werde und darum kein Grund zur Aufregung bestehe.

Es ist allgemein bekannt, dass Europa weder überwiegend für oder gegen die Türkei eingestellt ist, sondern die Mehrheit über keine Meinung im Hinblick auf die Türkei verfügt ${ }^{9}$. Dieses Klima der Ungewissheit kommt denen zugute, die die türkische Mitgliedschaft so weit wie möglich hinauszögern wollen.

\section{Die Herangehensweise der EU an die Türkei im Beitrittsprozess}

Die Türkei ist das einzige Land, das bereits vor dem Beitritt eine Zollunion mit der EU einging und diese seit Jahren fortsetzt. Nach dem Übergang zur Zollunion hat sich unser Außenhandelsdefizit mit Europa ungefähr verdoppelt. Während die Türkei diese Last trägt, nimmt sie den fünften Platz unter den Exportländern der EU ein. Die im Vertrag über die Zollunion der Türkei zugesicherte Unterstützung wurde zum größten Teil nicht verwirklicht ${ }^{10}$.

Heute werden Länder, die zu keinem Zeitpunkt einen vergleichbaren Beitrag zur EU geleistet haben, beinahe fraglos aufgenommen. Dennoch wird die Türkei, trotz ihrer mittel- und langfristig großen Vorteile, gezwungen, gegen den Strom zu schwimmen.

Europa muss endlich folgende Tatsache akzeptieren: Die türkische Mitgliedschaft in der EU ist für beide Seiten ohne Alternative. Diese Union ergibt sich zwingend aus Geographie, Geschichte und Politik. Eine Türkei, die außerhalb der EU bliebe, wird es wohl zu einem gewissen Grade schwerer haben, ihre Ziele zu erreichen. Für Europa aber gibt es keine Alternative, die den Raum der Türkei einnehmen könnte. Es wird darum im Interesse der EU sein, die Türkei zur EU-Mitgliedschaft zu ermutigen und sie bei der Erreichung der EU-Kriterien zu unterstützen.

Der Hauptakzent der heute alle politisch interessierten Kreise in Europa umfassenden Diskussion ist, ob die Grenzen Europas zukünftig geographisch, wirtschaftlich oder religiös/kulturell gezogen werden sollen. Die Türkei nimmt in dieser Diskussion, die Europa eigentlich vor Beginn des Erweiterungsprozesses hätte führen müssen, eine Position ein, die all diese Themen überspannt ${ }^{11}$. In Frage steht dabei letztlich grundsätzlich die Ausformung der europäischen Identität: Wird Europa in Zukunft nur eine christliche Identität tragen? Oder wird sie eine viele Religionen und Kulturen umfassende annehmen? Jedoch ist es einer EU, die sich selbst ausschließlich

9 Dr. Bahadır Kaleağası, Avrupa Yolunun Haritası, Istanbul 2003, S. 165.

10 Doç. Harun Gümrükçü, Türkiye ve $A B$, Istanbul 2002, S. 151.

11 Enis Çoșkun, Bütünleșme Sürecinde AB ve Türkiye, Istanbul 2001, S. 234-235. 
christlich definiert, unmöglich, die humanitären Werte zu bewahren, die die Europäische Union erst zu Europa machen.

Wenn sich Europa tatsächlich als »Europa der Werte« und als Zivilisationsprojekt versteht und sich dahin entwickeln will, wäre der dementsprechend erste zu unternehmende Schritt, die Türkei in die Mitgliedschaft aufzunehmen, die einzige funktionierende Demokratie in der islamischen Welt und zugleich das einzige muslimische Land, das mit seinem Rechtssystem und seinen Institutionen der westlichen Welt zugehört ${ }^{12}$.

Die türkische Mitgliedschaft wird dem Szenario eines »Krieges der Kulturen« entgegenwirken und durch den Beweis, dass Europa auf gemeinsamen Werten gegründet ist, die multikulturelle Struktur der EU stärken. Der Indikator dafür, dass der Westen den Islam nicht als Gefahr und als terroristische Kraft wahrnimmt, wird von der Entwicklung der Zusammenarbeit mit den muslimischen Ländern abhängen ${ }^{13}$. Die türkische Mitgliedschaft wird die These Huntingtons vom »Krieg der Kulturen«, die von den Regierungen offiziell zurückgewiesen, jedoch von breiten Teilen der Öffentlichkeit geteilt wird, entkräften. Nach dem 11. September besteht nicht nur seitens der EU und der USA, sondern der ganzen Welt dafür ein großer Bedarf.

Heute erkennt jeder an, dass der größte Erfolg der EU darin bestand, angefangen bei den Erzfeinden Deutschland und Frankreich, einen Krieg zwischen den Mitgliedsstaaten nicht nur undenkbar, sondern auch praktisch unmöglich zu machen ${ }^{14}$. Wenn dies aber das höchste Ziel und der größte Erfolg der EU war, könnte sie dann nicht auch einen Beitrag zur Verhinderung eines Krieges der Kulturen zwischen Kreuz und Halbmond leisten? Wäre es völlig unnötig, die Zukunft Europas auch gemäß einer solchen Mission zu formen?

Die Türkei, die mit ihrem historischen Erbe bewiesen hat, dass Menschen unterschiedlicher Kulturen und Religionen in Frieden miteinander leben können, will ihren Platz in der Familie der europäischen Völker finden. Auch Europa muss beweisen, dass es sich von seinen historischen Vorurteilen befreit hat und zum Architekten eines neuen Kulturprojektes geworden ist. Darum darf Europa die Hand der Türkei, gereicht, um Partner dieses ehrenvollen Projektes zu sein, nicht zurückweisen.

$\mathrm{Zu}$ den in Europa gegen die Mitgliedschaft der Türkei vorgebrachten Argumenten gehört ihre große Bevölkerungszahl; wirtschaftliche Probleme; dass sie die EU-Kriterien zu Demokratie und Menschenrechten noch nicht erfüllt habe, sie zumindest jedoch noch nicht in der Praxis verwirklicht sind; das Land physisch nicht zu Europa, sondern zu Asien gehöre; dass die Bevölkerungsmehrheit muslimisch sei und darum in einem religiösen Gegensatz zu Europa stünde oder auch dass sie auch kulturell nicht zu Europa gehöre. Im Grunde enthält jedes dieser Themen aber auch Fragen dazu, wie Europa seine eigene Zukunft gestalten will.

12 A. Nuri Yurdusev, Avrupa Kimliğinin Olușumu ve Türk Kimliği. Türkiye ve Avrupa, Ankara 1997, S. 69.

13 Dr. Yalçın Akdoğan, Kırk Yıllık Düss, Istanbul 2004, S. 33.

14 Enis Çoşkun, Türkiye-Avrupa Bütünleșmesinin Yüzylllık Seyir Defteri, Istanbul 2002, S. 61. 
Es ist erkennbar, dass Europa Probleme haben wird, die zum Beitrittszeitpunkt auf 70 Millionen geschätzte Bevölkerung der Türkei zu integrieren. Jedoch wird sich Europa in naher Zukunft aufgrund seiner alternden Bevölkerung gezwungen sehen, eine Verjüngungskur vorzunehmen. Mit dem Beitritt der Türkei werden die Probleme eines starken Zustroms von außen vermieden und die Verjüngung von innen vorgenommen. Insbesondere der ab dem Jahr 2010 zu erwartende Arbeitskräftemangel in Europa wird die Wichtigkeit der aus der Türkei kommenden Arbeitskraft weiter steigern $^{15}$.

Dass die türkische Wirtschaft schwerwiegende Probleme aufweist ist richtig. Aber in den vergangenen Jahren ist eine entschlossene Politik zur Überwindung dieser Probleme angewandt worden und es darf nicht übersehen werden, dass die Haushaltsdisziplin weitgehend hergestellt wurde. Auch ist offensichtlich, dass kein Europäer diesen riesigen Markt von 70 Millionen Menschen abschreiben möchte. Zudem ist die Türkei weder in politischer noch in wirtschaftlicher Hinsicht isoliert. Jenseits der Türkei erstrecken sich vom Nahen Osten bis nach Asien, vom Mittelmeer bis zum Schwarzen Meer ausgedehnte Territorien. Will Europa in diesen weiten Regionen nachhaltig wirksam sein, bedarf es der Partnerschaft der Türkei.

Es ist eine Tatsache, dass die Türkei im Hinblick auf Menschenrechte und Demokratie bisher den Standard der vorhandenen EU-Mitglieder nicht erreicht hat. Es ist jedoch auch eine Tatsache, dass eine Reihe von Ländern, die in naher Zukunft Mitglied werden, in dieser Hinsicht der Türkei nicht sehr voraus sind. Außerdem hat die Türkei seit dem Gipfel von Helsinki mit einer Reihe von Initiativen in diesem Zusammenhang ihre absolute Entschlossenheit gezeigt. Wenn man sich vor Augen führt, dass eine Reihe von europäischen Ländern, die nach den Terroranschlägen vom 11. September in den USA in Panik verfallen waren, Maßnahmen auf die Tagesordnung brachten, die die in der Türkei angewandte Praxis hinter sich ließen, wird die Bedeutung der in der Türkei unternommenen Schritte um so offensichtlicher. Und es darf auch nicht vergessen werden, dass die wichtige Triebkraft für die Türkei, weitere Fortschritte auf dem Feld von Demokratie und Menschenrechten zu unternehmen, die auf dem Weg in die EU zurückgelegte Strecke ist.

Die Behauptung derer, die die Türkei zu Asien rechnen, dass dann auch Russland, die Ukraine, Armenien, der Iran, Syrien, Israel, Palästina, Marokko oder gar die mittelasiatischen Staaten einen Anspruch auf EU-Mitgliedschaft geltend machen könnten, ist unlogisch, denn keines dieser Länder kann seinen Anspruch bis auf das Jahr 1959 zurückführen. Darum ist die Bewertung, dass, nur weil sich der größte Teil des türkischen Territoriums in Asien befindet, diese Länder in eine Position kämen, eine EU-Mitgliedschaft zu fordern, in keinster Weise schlüssig. Außerdem sollte man sich erinnern, dass die Orte, wo viele Philosophen, die Europa zu seinen kulturellen Grundlagen zählt, gelebt haben und die Europa als seine kulturelle und historische Wiege betrachtet, sich auf dem Territorium der Türkei befinden. Diejenigen, die die

15 Prof. Dr. Sedat Murat, AB ülkeleri ve Türkiye'nin karșılaștırmalı nüfus yapısı. Tüm Yönleriyle Türkiye-AB Ilișkileri, Istanbul 2002, S. 88. 
Türkei aufgrund ihrer geographischen Lage aus Europa ausgrenzen wollen, verleugnen in dieser Hinsicht eigentlich ihre historischen Wurzeln.

Der religiöse Unterschied gehört zu den stärksten Argumenten derjenigen, die die Türkei nicht in Europa sehen wollen. Dieses Thema jedoch bringt die Diskussion mit sich, ob sich Europa zukünftig christlich akzentuiert entwickeln wird und damit die überwunden geglaubte Intoleranz vergangener Jahrhunderte erneut die Herrschaft auf dem Kontinent antritt. Die Türkei ist der Auffassung, dass Europa, indem es sie in die Europäische Union mit ihrer mehrheitlich christlichen Bevölkerung aufnimmt, den Gipfel der Renaissance erreicht, die Europa erst zum modernen Europa machte. Die gegenteilige Entwicklung, d. h. die Zurückweisung der Türkei aufgrund der muslimischen Identität ihrer Bevölkerung ${ }^{16}$, wird Europa in die Zustände vor der Renaissance zurückwerfen.

\section{Die neue Vision und Mission, die die Türkei Europa gewinnt}

Europa hat sich bisher nicht entschieden, ob es eine globale Macht werden will. Die wirkungslose Haltung der EU in der Weltpolitik erweckt bei jedem Zweifel darüber, bis zu welchem Grade die Vision einer einheitlichen politischen Struktur der EU verwirklicht werden kann.

Während manche Kreise in der EU mit traditionellen Vorurteilen wie ihre muslimische Identität sowie ihrer anderen Kultur gegen die Mitgliedschaft der Türkei auftreten, wird übersehen, was die Türkei der EU (vor allem in Hinblick auf die Rolle, die sie in der Weltpolitik spielen könnte) gewinnen kann. Es lohnt nachzudenken, was für eine Rolle eine EU, die die Türkei zu ihren Mitgliedern zählt, bei der IrakKrise hätte spielen können und in welcher bestimmenden Position sie gewesen wäre. Ich bin überzeugt, dass der Verlauf der Irak-Krise die Gelegenheit geboten hat, festzustellen, dass es ein Fehler der EU war, die Mitgliedschaft der Türkei hinauszuzögern und zu verspäten.

Trotz aller erlebten unbefriedigenden Entwicklungen hat die EU die Chance noch nicht verloren, sich zur stärksten und wirksamsten Kraft des 21. Jahrhunderts zu entwickeln. Darum müssen jedoch neben der Vermeidung der Wiederholung vergangener Fehler die Entschlossenheit, eine solche Macht zu werden, offen gezeigt und die diesbezüglichen Schritte mit Mut und Geschwindigkeit eingeleitet werden.

Sowohl das in Jugoslawien Erlebte als auch die Irak-Krise haben gezeigt, dass die Staaten in der EU und die EU-Organe, statt des Schutzes kurzfristiger Berechnungen und Gleichgewichte, das Denken in größeren Maßstäben, die Entwicklung einer globalen Politik und in ihrer Verwirklichung absolute Entschlossenheit zeigen müssen.

Der Schritt, der die EU zur bestimmenden Kraft des 21. Jahrhunderts machen wird, wird die Mitgliedschaft der Türkei sein. Durch den Beitritt der Türkei wird die EU zu einem globalen Akteur werden, der internationalen Entwicklungen leichter die gewünschte Richtung geben kann. Die geographische Lage der Türkei, ihre Beziehungen 
zu den Nachbarstaaten, der islamischen Welt, Russland und Mittelasien, werden der EU die Chance eröffnen, auf internationalen Plattformen stärker mitzureden.

Wenn die Türkei EU-Mitglied wird, werden sich Europa in den es äußerst interessierenden Krisengebieten auf dem Balkan, dem Nahen Osten, dem Kaukasus und Mittelasien neue Perspektiven eröffnen und wird es neue Verantwortung übernehmen können.

\section{Ergebnis}

Die Türkei ist historisch, kulturell und geographisch seit fast 700 Jahren ein Teil des europäischen Kontinents. Die moderne Türkei hat seit der Zeit Atatürks bewusst den Weg einer Vereinigung mit Europa gewählt und über lange Jahre einen Beitrag zur Stabilität in Europa geleistet. Die Türkei ist zugleich auch ein Land, das durch seine Geschichte bewiesen hat, dass Menschen unterschiedlicher Religionen und Kulturen friedlich miteinander leben können. Mit dieser historischen Erfahrung will die Türkei Mitglied der europäischen Völkerfamilie werden.

Man wird Europa zwingen müssen, zu einer abschließenden Entscheidung in Sachen Türkei zu kommen. Solange man Europa nicht zu einer eindeutigen Haltung zwingt, wird sich die Türkei mit Ungewissheiten abplagen. Dieser Zeitpunkt ist jetzt gekommen. Im Jahr 2004 steht weniger für die Türkei, sondern eher die EU eine Prüfung an. 